\title{
65
}

\section{Assessing Internet Resources for Early Childhood Education}

Leonie Arthur, Bronwyn Beecher and Toni Downes

School of Education and Early Childhood Studies, University of Western Sydney, Penrith South 1797, Australia

l.arthur@uws.edu.au

Keywords: elementary education, evaluation, integration of ICT, internet, research

\begin{abstract}
Educators and parents must make sound educational decisions about of digital resources for use with young children. This paper reports on a project which evaluated current online resources for children eight years and younger. Over sixty sites were evaluated. Analysis examined the availability and quality of the sites and suitability for two groups: under fives and over fives. Many sites were difficult for young children to use independently and demanded expertise with literacy processes beyond the level of children in this age group.
\end{abstract}

\section{INTRODUCTION}

For many young Australian children desktop computers, DVDs, gameboys and mobile phones are a familiar aspect of their home and community environments. In many of these homes the computer is connected to the Internet. The Australian Bureau of Statistics (ABS) estimates that over $60 \%$ of Australian households with children have computers. For households with younger children, the percentages are slightly lower, with $48 \%$ of households with children aged between birth and four years and $54 \%$ of households with children aged between five and nine years having computers (ABS 1998). The picture with regard to the Internet also reveals significant access by young children. In 1998 approximately a third of a million households with young children had Internet connections (ABS 
1998). Between 1996 and 1998 the rate of Internet access increased four-fold (ABS 1998). Given these accelerated rates of Internet connections to homes, the 1998 figures are likely to be quite conservative. It would be reasonable to estimate that in 2000 over half of preschool age children in Australia have access to a computer in their home, and two thirds of these also have Internet access.

Many families in Australia are mindful that their children learn through interactions with digital resources. Families of preschool aged children in a large project that mapped early literacy practices in New South Wales Australia noted that their children learnt literacy early and fast and in enjoyable ways through technology (Makin, Hayden, Holland, Arthur, Beecher, Jones Diaz and McNaught 1999). Most parents interviewed (71\%) identified technology as important to their child's literacy learning especially for bilingual children. Similarly in the United States, families from a range of socio-economic backgrounds with children aged 2 to 17 years regarded Internet use as beneficial for children and very useful within the family for communicating and learning (National Schools Board Foundation 2000). In this study, families who connected to the Internet to support education found that their children's learning with the Internet went beyond schoolwork and that their attitude to educational settings improved. In addition, rather than interrupting children's healthy activities the Internet often actually promoted less television watching and encouraged more interests, increasing time playing outdoors as well as involvement in creative arts. The Internet was also seen as promoting communication between families, children and others. Families viewed the Internet as providing potentials for improved communication with their child's teacher and educational setting about their child's learning as well as better access to management processes.

In general, these positive attitudes and patterns of access to computers and the Internet do not flow over to early childhood settings. Currently in day care centres and preschools in Australia there are low levels of computer use and almost no Internet use (Makin et al. 1999). While there are a range of complex factors that help explain such low use, educators' attitudes and perceptions about the appropriateness of access to and use of computers and the Internet play a significant part (Dockett, Perry and Nanlohy 1999).

Amongst early childhood educators there is much debate about the appropriateness of access to and use of the Internet with young children under eight. Educators are often resistant to incorporating technology in early childhood programs (McNaught, Clugston, Arthur, Beecher, Ashton, Hayden and Makin 2000). The language that many early childhood educators use about computers indicates that they have constructed 'using the computer' as an activity separate from and in opposition to social interaction and appropriate play experiences. It is as if the spectre of 'solo, intense 
computer game-playing' over-rides all other possibilities in the minds of the educators.

These prevailing negative attitudes are often difficult to change even with the evidence of long term research related to the beneficial effects of developmentally appropriate computer use as well as parents' anecdotal evidence of children's learning at home with computers. Appropriate professional development can assist in the process of attitudinal change and thus changes in practices (McNaught et al. 2000).

However, professional development while necessary is not sufficient. Early childhood educators need access to appropriate quality digital resources. This includes reliable hardware, quality software, relatively cheap and fast access to the Internet and quality online resources. Furthermore they need an understandable framework from which they can generate learning activities using these quality digital resources.

One way of framing the pedagogy and curriculum around use of digital resources is to consider these resources in parallel to other resources already found in early childhood settings, such as paint, blocks, books and sand pits (Downes, Beecher and Arthur 2000). The task of the early childhood educator, then, becomes one of structuring learning environments in which young children use digital media and tools to express and represent their thoughts, understandings and feelings as they make sense of their worlds just as they would with traditional media (paper, sand, blocks) and tools (brushes and spades). All these resources offer children alternate ways to play, interact with others, investigate their environments, to solve problems and to be creative.

Positive learning outcomes require educators to make pedagogically sound decisions about the nature of the learning experience and the appropriateness of the digital resource. Teachers and parents need to pay attention to evaluating digital resources in the same way as they would for other learning resources. This paper reports on a project where current online resources were evaluated for use with young children.

\section{OVERVIEW OF PROJECT}

The project reported upon in this paper was conducted in 1999. It focused on the use of digital resources, particularly the Internet, with children eight years and under. Participants in the study represented the stakeholders in early childhood education across day care, preschool and early years of school: educators, systems representatives, academics, researchers, policy makers and advisers, parents and children. They were asked to reflect on their beliefs and practices with digital technologies and to suggest ways in 
which Internet sites could be better designed and used to support children's learning.

Data concerning the participants' views and current practices involving digital resources were collected in a range of ways. Methods included: a one day national workshop for early childhood educators and researchers from around Australia; telephone interviews with early childhood educators; focus group discussions with parents and with children; observations of children interacting with online resources; and evaluation of online educational and recreational sites. Early childhood teacher education students identified and evaluated appropriate educational, recreational and popular culture sites relevant and appropriate for young children in the age groups birth to five and five to eight and they identified key elements in the nature and provision of Internet sites for young children.

Children's sites on the Internet were judged according to the nature, safety, portability and the overall quality of the site with consideration given to the site's purpose. Quality was defined in terms of content and design.

\subsection{Content}

Five key criteria were used to assess quality.

\subsubsection{Open-ended experiences}

Resources that are open-ended enable children to respond and interact in their individual ways, at their own pace, and to explore their own interests. Thus children can investigate, problem solve and jointly construct with others as they pose questions and share findings. In doing so, children often discover new unintended uses for resources and become highly engaged in their investigations.

\subsubsection{Appropriate challenges}

Online resources for young children need to respond to what children can do independently as well as what children can do with assistance from peers and adults. The nature of complexities inherent in the resource needs to be appropriate to the child in addition to being culturally relevant and of personal interest. Appropriate resources move with the child's changing competencies and learnings (NAEYC 1998) and can thus be used repeatedly by the child (Mamamedia 1998). 


\subsubsection{Opportunities for collaboration}

Useful online resources for young children promote social interactions, contributing to increased levels of co-operation and assistance. Children prefer to collaborate with one or two other children to working alone (NAEYC 1998). Associated with this collaboration is often language learning as children discuss ideas, take turns and negotiate. The Internet also provides children with opportunities for collaboration with others beyond the setting and so broadens a narrow, local or ethnocentric view of people and the world.

\subsubsection{Quality of information and experiences}

Children benefit from access to sites that offer an expansive range of information and experiences that are relevant to their own lives and interests as well as to the curricula of the first years of school. The information needs to be accurate and current and to be organised and presented in ways that reflect various mixes and levels of challenge of aural, visual and written texts.

\subsubsection{Ability to cater for diversity and challenge stereotypes}

All resources convey explicit and implicit messages about people to children. Each child's uniqueness should be acknowledged and supported by positive sounds, images and texts about cultures, languages, lifestyles, families and differing abilities (NAEYC 1998). In addition, opportunities to challenge and critique prevailing assumptions and stereotypes are important for children from the dominant culture as well as for those from minorities.

\subsection{Design}

Four key criteria were used to assess the quality of design.

\subsubsection{Integration of sound, text, graphics and animation}

Sites attractive to young children appeal to their senses, since this is how children explore and learn about their environment. Children want to investigate and seek to explore objects that respond in some way to their actions. Therefore appearance, sound and animation are significant aspects to consider when evaluating the suitability of a resource. Integrated sound, graphics, animation and text are necessary within resources, especially for under fives (Mamamedia 1998, De Voogd and Kritt 1997). 


\subsubsection{Ease of navigation}

Sites need to be easy to access and to use. Navigation within the site and between the various activities needs to be obviously different to that which takes the child into and out of the site, or from the activities to the marketing areas. Consistency in layout promotes children's successful navigation (ElTigi 1996).

\subsubsection{Efficient technology}

Children want resources to respond quickly to their actions, continue the interactions and propose some form of feedback. Sites need to be stable, consistently available and load with ease. Young children's requirements for resources offering quality graphics, sound, text and speed contradict what technology can presently supply. The purpose of these features needs to be assessed by educators when making judgements about efficient technology of resources.

\subsubsection{Safety}

Safety is of prime importance as children's contact with Internet resources increases and commercial sites multiply. Families express concerns about children being targeted and used for commercial purposes and seek security assurances. Current debates include claims of deceptive online marketing where children are targeted by advertisers, where content and advertising are integrated and where characters promote products (see http://www.cme.org). Implications for judging sites include:

- Checking that there is a clear distinction between advertising and editorial focus;

- Ensuring that the family need to give permission before information is collected from children; and

- Checking that the privacy policy declares if collected information is sold to unknown parties (Mamamedia 1998).

\section{FINDINGS}

The analysis of current online resources for young children identified a growing number of sites that were labelled as children's sites. Over sixty sites for young children were located and evaluated. Children's sites on the Internet can be grouped into four types based on the nature of the activity undertaken. There were design and make sites, work and play sites 
(including activity and game, explore and investigate, and information sites), communicate and share sites, and online project sites. While these groups are not mutually exclusive, they provide a useful way of thinking about the nature and quality of Internet sites for young children.

\subsection{Analysis of online resources}

There was significant variation in the number and quality of sites suitable for different age groups. Similarly, there were variations, in number and quality of the various types of sites. Each of these differences will be discussed below.

\subsubsection{Sites for the under fives and over fives}

Overall the range and quality of resources for independent use by children under five were poor. Currently the higher quality digital resources that are appropriate for independent use by children of this age group already exist as CD-ROM based software. Many Internet sites required physical manipulation that meant it was difficult for children under five to navigate their way around. The sites were also heavily reliant on print text to make meaning. Most Internet experiences for this age group will continue to be useful in the short to medium term, when they are mediated, if only in part, by older peers, parents and educators. Communication and sharing sites, in particular, will remain joint activities until voice recognition and transmission are better-developed technologies.

For the five to eight year olds, the quality and range of resources were patchy, yet there were sufficient sites of quality to enable educators working in the early years of school to effectively integrate digital resources from the Internet into their curriculum. Of uniform quality were the online project sites, both in terms of the range of sites offered and the quality of the actual projects. Design and make sites could not compete with their CD-ROM counterparts. Appropriate work and play sites, particularly information sites, while patchy in quality, existed in sufficient numbers to make a validated collected of quality sites viable and extremely useful to educators. Communicate and share sites may continue to have little place in educational settings for their roles are already fulfilled by email and online project sites.

\subsubsection{Availability and quality of different types of sites}

There were few design and make sites which offered children the opportunity to create, make, design or build. The few sites for under fives reflected closed experiences, such as puzzles and colouring in, whilst sites 
for five to eight year olds offered more active and open-ended experiences such as making postcards and writing letters. However, these experiences demanded expertise with literacy processes beyond the level of many children in this age group.

Three types of work and play sites emerged:

- Activity and game sites: Many of these offered closed and trivial experiences which provided limited opportunities for making meaning and developing understandings. However, children often found these sites interesting and used them at home as they linked to information sites about popular culture characters.

- Investigating sites: There were very few sites offering investigative experiences. No such sites were found for children under five and few for five to eight year olds. The sites that were available, such as 'educational' sites provided by museums, more often than not provided closed experiences such as colouring.

- Information sites: The Internet provides easy access to information in relation to leisure activities and for school-related purposes. It was found that information sites abound for school curriculum areas and topics but rarely did these collections provide pointers to sites for under fives nor did similar validated collections of information sites exist for the under fives. Some sites appeared at first to be appropriate for under fives although the actual information the children were taken to was more appropriate for older children. The content of the information sites evaluated, however, would be valuable as resources for adult-directed or scaffolded use.

The Internet was seen by parents who participated in the consultation to be most useful as a tool for communicating with others although early childhood educators rarely spoke of using the Internet for themselves or with children in this way. As well the basic functions such as e-mail, the Internet provides a range of sites where children can chat with each other in a 'safe environment' and where they can publish their writings and artwork. However these sites seemed more appropriate for home than for educational settings, in the sense that e-mail (to known recipients) and communication and sharing through online projects already provide these facilities for educational settings.

Online project sites were mostly provided by educational authorities or organisations and included a range of high quality projects in which early childhood educators and young children could participate. The projects were aimed at the early years of school, although they could be adapted for use with younger children. While the projects tended to be structured, they encouraged high degrees of sharing and included possibilities for open- 
ended experiences. However, these sites were not generally used by early childhood educators.

\section{CONCLUSIONS}

Overall, the quality of sites that would be appropriate for young children to use independently was poor or patchy, with a better range being available for the five to eight year olds. However, many of the quality sites that had content suitable for younger children but required the reading of text would be appropriate for young children with adult assistance.

In terms of quality across various types of sites, the findings were mixed. The commercial activity and games sites, while well-designed, offered limited opportunity for making meaning or being creative. Most of the activities on work and play sites were closed and trivial. Greater potential for appropriate open-ended activities lies with the communicate-and-share sites and the specially designed online project sites. However, these types of sites were rarely accessed from home (where connection to the Internet was more common) or the early childhood centres. It would be fair to say that in general the CD-ROM based software currently offers the wider range of high quality open-ended learning environments for young children to use independently.

However, given the rapid expansion of the Internet in terms of access and the increase in the number and quality of sites, parents, educators, curriculum and policy makers need to become more aware of the potential of quality sites to enhance recreational and educational activities for young children, and to use these sites as they become more readily accessible.

\section{REFERENCES}

ABS (Australian Bureau of Statistics) (1998) Household use of information technology (Australian Catalogue No 8146.0). Commonwealth of Australia, Canberra.

Clements, D. (1999) Young children and technology. In Dialogue on early childhood science, mathematics and technology education, G. D. Nelson (Ed.), pp. 92-105. American Association for the Advancement of Science, Washington, DC.

DeVoogd, G. and Kritt, D. (1997) Computer mediated instruction for young children: Teachers and software missing the zone. Site 97-Young children. University of New Orleans, New Orleans.

Dockett, S., Perry, B. and Nanlohy, P. (1999) Computers in early childhood services: A part of the educational program or less time to play? ARECE 99 Computers in Early Childhood Services.

Downes, T., Beecher, B. and Arthur. L. (2000) Effective learning environments for young children using digital resources: An Australian perspective. A paper presented at the 
World Congress on Computers in Beijing, China, August 2000 (contact t.downes@uws.edu.au for a copy of the paper).

El-Tigi, M. A. (1996) Perception of elementary students of visuals on the web. VisionQuest: Journeys towards visual literacy. Selected readings from the annual conference of the International Visual Literacy Association.

Mamamedia. (1998) What makes a good kids' website?

[http://mamamedia.com/area/grownups/newhome.html].

Makin, L., Hayden, J., Holland, A., Arthur, L., Beecher, B., Jones Diaz, C. and McNaught, M. (1999) Mapping literacy practices in early childhood settings. $2^{\text {nd }}$ edition, NSW

Department of Community Services and NSW Department of Education and Training,Sydney.

McNaught, M., Clugston, L., Arthur, L., Beecher, B., Ashton, J., Hayden, J. and Makin. L. (2000) The early literacy and social justice project: Final report. NSW Department of Education and Training and NSW Department of Community Services, Sydney.

NAEYC (National Association for the Education of Young Children) (1998) Position Statement on Technology and Young Children [http://www.naeyc.org/about/position/pstech98.htm]

National Schools Board Foundation (2000) Safe and smart: Research guidelines for children's use of the internet. [http://www.nsbf.org/safe-smart/full-report.htm].

Roberts, D. F., Foehr, U. G., Rideout, V. J. and Brodie, M. (1999) Kids and media @ the new millennium. A Kaiser Family Foundation Report.

\section{BIOGRAPHY}

Leonie Arthur lectures in early childhood education; her research and teaching interests are in the areas of children's literacy including the role of play in literacy learning and multiple literacies. Bronwyn Beecher lectures in early literacy learning; her main research and teaching areas include links between prior to school and school settings, the role of technologies in literacy learning and literacy play. Toni Downes is an Associate Professor in educational computing; her current research projects span from early childhood education to university education. 